\title{
One-loop correction to the photon velocity in Lorentz-violating QED
}

\author{
Petr Satunin \\ Institute for Nuclear Research of the Russian Academy of Sciences, \\ 60th October Anniversary Prospect, 7a, 117312 Moscow, Russia
}

(Received 29 November 2017; published 20 June 2018)

\begin{abstract}
We calculate a finite momentum-dependent part of the photon polarization operator in a simple model of Lorentz-violating quantum electrodynamics nonperturbatively at all orders of Lorentz-violating parameters. We sum one-particle reducible diagrams into the modified photon propagator and determine the physical photon dispersion relation as the location of its pole. The photon dispersion relation, as well as its group velocity, acquires the one-loop momentum-dependent radiative correction. We constrain the Lorentz-violating parameters for heavy charged fermions (muon, $\tau$ lepton, and top quark) from the photon timing observations.
\end{abstract}

DOI: 10.1103/PhysRevD.97.125016

\section{INTRODUCTION}

Small violation of Lorentz invariance (LI) may take place in physics at low energies as a relic of some unknown ultraviolet theory, which includes quantum gravity. There are numerous approaches to quantum gravity, featuring Lorentz invariance violation (LV), such as loop quantum gravity [1], noncommutative field theory [2], spacetime foam [3], some approaches in string theory [4], HoravaLifshitz gravity [5,6], and others (see Refs. [7-9] for reviews). In accordance with these models, small but nonzero LV may appear in the matter sector as well.

Lorentz invariance violation in the matter sector may be also considered phenomenologically. The most general framework describing LV in the matter sector is called the Standard Model extension (SME) [10]. The SME Lagrangian includes all possible operators of a given order, which are scalars under coordinate transformations. These operators are controlled by the coefficients which may be tested (and constrained) experimentally (see Ref. [11]).

Quantum field theory methods in a theory without LI may be developed in analogy with corresponding LI theory. Such models can be quantized [12], and Feynman rules for perturbative calculations can be derived, for full SME or a certain sector of it $[13,14]$. Tree-level processes in this type of models are deeply investigated. Several processes, kinematically forbidden in LI theory, may occur in its LV extension; thresholds and cross sections of other processes may be modified [13-16]. These tree-level

\footnotetext{
*satunin@ms2.inr.ac.ru
}

Published by the American Physical Society under the terms of the Creative Commons Attribution 4.0 International license. Further distribution of this work must maintain attribution to the author(s) and the published article's title, journal citation, and DOI. Funded by SCOAP. phenomena lead to several experimental constraints on SME parameters [11].

Loop-level processes in theories without LI have been also studied since Ref. [17]. One of the main achievements in this area is the proof of one-loop renormalizability of the QED sector of the SME [18]. In that work, infinite parts of one-loop diagrams have been calculated. A study of finite radiative corrections was initiated in Ref. [19]. The authors of Ref. [19] have shown that these corrections may be momentum dependent and may influence the propagation of free particles (see also Ref. [20]).

The last statement can be illustrated in the following way. A chain of one-particle reducible diagrams (polarization operators or self-energy) can be summed into the one-loop modified propagator. The poles of the propagator determine the modified dispersion relation for the corresponding particle. In the LI case, the corrections to the denominator of the propagator reduce to the renormalization of fields and parameters, but in the LV case, they do not. There are two examples in standard physics: photon dispersion in external magnetic (see e.g., Ref. [21]) and gravitational [22] fields. Both external classical fields violate LI, and in both cases, photon velocity depends on its energy as well as on the external field parameters. The similar situation may occur if LI is broken at fundamental level like in the SME.

Cambiaso et al. [19] have calculated the momentumdependent radiative correction to the electron dispersion relation in a leading order on SME parameters in a simplified $C P T$-even nonbirefringent version of the SME. They used a general technique of calculations in the SME-perturbative treatment of LV parameters. However, in the most simplified models, which are both $C P T$ even and isotropic, nonperturbative treatment can be possible. Thus, both external states and propagators may be computed exactly on LV parameters. This allows us to use 
these expressions to compute loop diagrams nonperturbatively at all orders in LV parameters. The aim of this article is to adopt this nonperturbative approach in the calculation of the finite momentum-dependent part of the photon polarization operator, which allows us to calculate one-loop radiative correction to the photon dispersion relation. The charge of this nonperturbative treatment is the restriction to a very limited number of $L V$ parameters.

The paper is organized as follows. In Sec. II, we describe a simplified model that we use for calculations. In Sec. III, we provide the one-loop calculation of the photon polarization operator, summarize one-loop radiative corrections to the photon propagator, and compute the modified photon dispersion relation. In Sec. IV, we establish bounds on LV parameters for charged fermions from photon timing observations. Section V is devoted to a discussion.

\section{MODEL}

The QED sector of SME is described by the Lagrangian [10]

$$
\begin{aligned}
\mathcal{L}_{\mathrm{SME}}= & -\frac{1}{4} F_{\mu \nu} F^{\mu \nu}+i \bar{\psi} \Gamma^{\mu} D_{\mu} \psi-\bar{\psi} M \psi \\
& -\frac{1}{4}\left(k_{F}\right)_{\mu \nu \rho \sigma} F^{\mu \nu} F^{\rho \sigma}+\frac{1}{2}\left(k_{A F}\right)^{\kappa} \epsilon_{\kappa \lambda \mu \nu} A^{\lambda} F^{\mu \nu},
\end{aligned}
$$

where the constants $\left(k_{F}\right)_{\mu \nu \rho \sigma},\left(k_{A F}\right)^{\mu}$ control LV in photon sector and $\Gamma^{\mu}$ and $M$ have the following form:

$\Gamma^{\mu}=\gamma^{\mu}+c^{\mu \nu} \gamma_{\nu}+d^{\mu \nu} \gamma_{5} \gamma_{\nu}+i f^{\mu}+\frac{1}{2} g^{\lambda \nu \mu} \sigma_{\lambda \nu}+e^{\mu}$,

$M=m+a^{\mu} \gamma_{\mu}+b^{\mu} \gamma_{5} \gamma_{\mu}+\frac{1}{2} H^{\mu \nu} \sigma_{\mu \nu}$.

Here, $a^{\mu}, b^{\mu}, c^{\mu \nu}, d^{\mu \nu}, e^{\mu}, f^{\mu}, g^{\lambda \mu \nu}$, and $H^{\mu \nu}$ are the constants controlling all types of Lorentz and $C P T$ violation. The electromagnetic field strength $F_{\mu \nu} \equiv \partial_{\mu} A_{\nu}-\partial_{\nu} A_{\mu}$ and the covariant derivative $D_{\mu}=\partial_{\mu}-i e A_{\mu}$ are defined in a usual way, and $e$ is the electron charge. The greek indices $\mu, \nu \ldots$ are raised and lowered by using the Minkowski metric. It was shown that the Lagrangian (1) is one-loop renormalizable [18] and does not lose renormalizability in curved spacetime [23].

Note that the renormalization procedure [18] implies a certain mixing of some coefficients in (2). Nevertheless, there are several separate sectors in the parametric space which are compatible with the structure of SME renormalization, for example the $C$ - and $C P T$-even sectors in which all coefficients except $\left(k_{F}\right)_{\mu \nu \rho \sigma}$ and $c_{\mu \nu}$ are set to zero. ${ }^{1}$ In this work, we restrict ourselves to the $\mathrm{SO}(3)$-invariant sector in which all time components of $\left(k_{F}\right)_{\mu \nu \rho \sigma}$ and $c_{\mu \nu}$ are set to zero

\footnotetext{
${ }^{1}$ This model, with the reduced form of $\left(k_{F}\right)_{\mu \nu \rho \sigma}$, has been considered in Ref. [19].
}

while each space component is characterized by a single parameter:

$$
\left(k_{F}\right)_{\mu \nu \rho \sigma}=c_{\gamma} \cdot \delta_{\mu}^{i} \delta_{\nu}^{j} \delta_{\rho}^{k} \delta_{\sigma}^{l}\left(\delta_{i k} \delta_{j l}-\delta_{i l} \delta_{j k}\right), \quad c_{\mu \nu}=c_{e} \cdot \delta_{\mu}^{i} \delta_{\nu}^{j} \delta_{i j} .
$$

The greek indices $\mu, \nu \ldots$ run from 0 to 3 , while the latin indices $i, j \ldots$ are spacelike, taking the values $1,2,3$. Substituting the expressions (3) into the Lagrangian (1), we obtain the following model:

$$
\begin{aligned}
\mathcal{L}_{\text {model }}= & -\frac{1}{4} F_{\mu \nu} F^{\mu \nu}+i \bar{\psi} \gamma^{\mu} D_{\mu} \psi-m \bar{\psi} \psi \\
& -\frac{c_{\gamma}}{2} F_{i j} F^{i j}-i c_{e} \bar{\psi} \gamma^{i} D_{i} \psi .
\end{aligned}
$$

Here, the parameters $c_{\gamma}$ and $c_{e}$ determine the maximal velocities for the photon and electron/positron respectively. Namely, the Lagrangian (4) yields the following tree-level dispersion relations:

$$
\begin{aligned}
\gamma: k_{0}^{2} & =\left(1+c_{\gamma}\right)^{2} \vec{k}^{2} \simeq\left(1+2 c_{\gamma}\right) \vec{k}^{2}, \\
e^{ \pm}: E^{2} & =\left(1+c_{e}\right)^{2} p^{2}+m^{2} \simeq\left(1+2 c_{e}\right) \vec{k}^{2}+m^{2} .
\end{aligned}
$$

Let us notice that one can redefine fields and coordinates in such a way that one of the parameters $\left(c_{e}\right.$ and $\left.c_{\gamma}\right)$ disappears from the Lagrangian (4), since only the difference $\left(c_{e}-c_{\gamma}\right)$ carries physical meaning. ${ }^{2}$ However, for completeness, we will keep both of them for further analysis.

The model (4) has been described in Ref. [24], where the exact expression for external states and propagators has been obtained. The electron propagator has the same form as in the standard case,

$$
S(p)=\frac{i\left(\gamma^{\mu} \hat{p}_{\mu}-m\right)}{\hat{p}^{2}-m^{2}},
$$

where $\hat{p}_{\mu}$ is no longer the electron four-momentum but $\hat{p}_{\mu}=\left(p_{0},\left(1+c_{e}\right) p_{i}\right), \hat{p}^{2}=\hat{p}_{\mu} \hat{p}^{\mu}$. In order to write the photon propagator, one should first add to the Lagrangian (4) a gauge-fixing term, which, in order to get rid of nondiagonal terms in propagator, is convenient to take in the form

$$
\mathcal{L}_{g f}=-\frac{1-2 c_{\gamma}}{2}\left(\partial_{0} A_{0}-\left(1+2 c_{\gamma}\right) \partial_{i} A_{i}\right)^{2} .
$$

\footnotetext{
${ }^{2}$ In particular, redefinitions $x_{i} \rightarrow\left(1+c_{e}\right) x_{i}, A_{i} \rightarrow\left(1+c_{e}\right)^{-1} A_{i}$, $\psi \rightarrow\left(1+c_{e}\right)^{3 / 2} \psi$ in the action $S=\int d^{4} z \mathcal{L}_{\text {model }}$ remove the parameter $c_{e}$ from the fermionic part of the model. In terms of these new fields, the combination $\left(c_{\gamma}-c_{e}\right)$ appears in the photon dispersion relation (5) instead of $c_{\gamma}$.
} 
Inverting the quadratic part of the photon Lagrangian in (4) with (8), one obtains the photon propagator (cf. Refs. [14,24]) in "pseudo-Lorentz" gauge:

$$
D^{\mu \nu}(k)=-i \frac{\operatorname{diag}\left(\left(1+2 c_{\gamma}\right),-1,-1,-1\right)}{k_{0}^{2}-\left(1+2 c_{\gamma}\right) \vec{k}^{2}} .
$$

For our calculation, the photon propagator in the Coulomb gauge $\partial_{i} A_{i}=0$ is more convenient:

$$
\begin{aligned}
D^{00}(k) & =i \frac{1}{\left(1+2 c_{\gamma}\right) \vec{k}^{2}}, \quad D^{0 i}(k)=0, \\
D^{i j}(k) & =i \frac{\delta^{i j}-\frac{k^{i} k^{j}}{\vec{k}^{2}}}{k_{0}^{2}-\left(1+2 c_{\gamma}\right) \vec{k}^{2}} .
\end{aligned}
$$

To obtain the polarization operator, one also needs to know the photon-fermion vertex, which now takes the form

$$
\Gamma_{\mu}=-i e\left(\gamma_{0},\left(1+c_{e}\right) \gamma_{i}\right) .
$$

We apply the expressions (7), (9)-(11) to compute the oneloop photon polarization operator.

\section{PHOTON POLARIZATION OPERATOR}

The aim of this section is to calculate the photon polarization operator in the one-loop approximation and subsequently resum one-loop contributions into the photon propagator.

Following the rules of the standard perturbation theory, let us write the expression for the photon polarization operator in the one-loop approximation,

$$
\Pi_{\mu \nu}(k)=\int \frac{d^{4} k_{l}}{(2 \pi)^{4}} \operatorname{Tr}\left[\Gamma_{\mu} S\left(k+k_{l}\right) \Gamma_{\nu} S\left(k_{l}\right)\right],
$$

where $\Gamma_{\mu}$ and $S\left(k_{l}\right)$ are the vertex and propagator defined in the previous section and $k_{l}$ is the loop momentum. Rescaling the loop momentum, $k_{l}^{i} \rightarrow\left(1+c_{e}\right) k_{l}^{i}$, and introducing the "hat" momentum, $\hat{k}=\left(k_{0},\left(1+c_{e}\right) k_{i}\right)$, we can represent the components $\Pi_{00}, \Pi_{0 i}$, and $\Pi_{i j}$ of the photon polarization operator (12) via LI ones:

$$
\begin{aligned}
\Pi_{00}(k) & =\left(1+c_{e}\right)^{-3} \Pi_{00}^{L I}(\hat{k}), \\
\Pi_{0 i}(k) & =\left(1+c_{e}\right)^{-2} \Pi_{0 i}^{L I}(\hat{k}), \\
\Pi_{i j}(k) & =\left(1+c_{e}\right)^{-1} \Pi_{i j}^{L I}(\hat{k}) .
\end{aligned}
$$

Here, $\Pi_{\mu \nu}^{L I}(k)$ is the standard LI polarization operator

$$
\Pi_{\mu \nu}^{L I}(k)=\left(\eta_{\mu \nu}-\frac{k_{\mu} k_{\nu}}{k^{2}}\right) k^{2} \Pi\left(k^{2}\right),
$$

where $\Pi\left(k^{2}\right)$ is expressed via the dimensional regularization technique as

$$
\begin{aligned}
\Pi\left(k^{2}\right)= & -\frac{e^{2}}{2 \pi^{2}} \int_{0}^{1} d x x(1-x) \\
& \times\left[\frac{1}{\varepsilon}+\ln 4 \pi-\gamma_{E}-\ln \frac{m^{2}-x(1-x) k^{2}}{\mu^{2}}\right] .
\end{aligned}
$$

Here, $\varepsilon=4-2 d$ tends to zero as the number of dimensions $d$ tends to $4, \gamma_{E}$ is the Euler constant. By using Eqs. (13)-(15), we rewrite the LV polarization operator as follows,

$$
\Pi_{\mu \nu}(k)=\left[\left(1-c_{e}\right) k^{2}\left(P_{1}\right)_{\mu \nu}-2 c_{e} \vec{k}^{2}\left(P_{2}\right)_{\mu \nu}\right] \Pi\left(\hat{k}^{2}\right),
$$

where we have introduced two projection operators,

$$
P_{1}^{\mu \nu}=\eta^{\mu \nu}-\frac{k^{\mu} k^{\nu}}{k^{2}}, \quad P_{2}^{\mu \nu}=-\delta_{i}^{\mu} \delta_{j}^{\nu}\left(\delta^{i j}-\frac{k^{i} k^{j}}{\vec{k}^{2}}\right),
$$

with the properties $P_{1 \nu}^{\mu} P_{1 \lambda}^{\nu}=P_{1 \lambda}^{\mu}, \quad P_{2 \nu}^{\mu} P_{2 \lambda}^{\nu}=P_{2 \lambda}^{\mu}$, $P_{1 \nu}^{\mu} P_{2 \lambda}^{\nu}=P_{2 \lambda}^{\mu}$, and

$\Pi\left(\hat{k}^{2}\right)=\frac{e^{2}}{2 \pi^{2}} \int_{0}^{1} d x x(1-x) \ln \left(1-x(1-x) \frac{\hat{k}^{2}}{m^{2}}\right)+C_{\Pi}$,

where $C_{\Pi}=-\frac{e^{2}}{12 \pi^{2}}\left(\frac{1}{\varepsilon}+\ln 4 \pi-\gamma_{E}-\ln \frac{m^{2}}{\mu^{2}}\right)$.

The polarization operator (16) contains two infinite terms proportional to the projectors $P_{1}^{\mu \nu}$ and $P_{2}^{\mu \nu}$. In the renormalization procedure, they are contracted with their counterterms appearing from renormalizing the electromagnetic field $A_{\mu}$ and parameter $c_{\gamma}$ respectively (see Ref. [18] for detailed calculations in the SME). Dependent on the concrete subtraction scheme, renormalized constant $C_{\Pi}$ may take different values. Being interested in the propagation of a free photon, we apply the on-shell subtraction scheme. From physical grounds, we assume no radiative corrections for soft on-shell photons. This can be achieved by setting $C_{\Pi}=0$, which results in $\Pi(0)=0$.

Let us sum a chain of one-particle reducible diagrams into the modified photon propagator. This procedure is simpler if we take photon propagator in Coulomb gauge (10) (see the Appendix for comparison with pseudoLorentz gauge). The summation goes independently for the time and space parts and leads to the result [cf. (10)]

$$
\begin{aligned}
D_{1-\text { loop }}^{00}(k)= & \frac{i}{\vec{k}^{2}\left(1+2 c_{\gamma}-\left(1-c_{e}\right) \Pi\left(\hat{k}^{2}\right)\right)}, \\
D_{1-\text { loop }}^{0 i}(k)= & 0, \\
D_{1-\text { loop }}^{i j}(k)= & \frac{i}{1-\Pi\left(\hat{k}^{2}\right)\left(1-c_{e}\right)} \\
& \cdot \frac{\delta^{i j}-\frac{k^{i} k^{j}}{\vec{k}^{2}}}{k_{0}^{2}-\vec{k}^{2}\left(1+2 c_{\gamma}+2\left(c_{\gamma}-c_{e}\right) \Pi\left(\hat{k}^{2}\right)\right)} .
\end{aligned}
$$


Time components of the propagator, $D^{00}$ and $D^{0 i}$, are the same as in the tree-level propagator (up to the coefficient), and the space component $D^{i j}$ keeps its tensor structure proportional to the projector $P_{2}^{\mu \nu}$, but the pole structure of the denominator changes. It is known that the position of the pole in (20) determines the dispersion relation for a free photon. The pole from the first term, $1 /\left[1-\Pi\left(\hat{k}^{2}\right)\left(1-c_{e}\right)\right]$, is a usual Landau pole. The pole from the second term of (20) is physical. Hence, to find the photon dispersion relation explicitly, one should solve the equation

$$
k_{0}^{2}-\vec{k}^{2}\left(1+2 c_{\gamma}+2\left(c_{\gamma}-c_{e}\right) \Pi\left(\hat{k}^{2}\right)\right)=0,
$$

where $\Pi\left(\hat{k}^{2}\right)$, given by (18), includes the zeroth component of the momentum $k_{0}$ as well. For this purpose, we apply an iteration procedure: we start from the tree-level dispersion relation (5) at zero order and consider $\Pi\left(\hat{k}^{2}\right)$ as a small perturbation suppressed by $\alpha_{e m}=e^{2} / 4 \pi$. At the first order on $\alpha_{e m}$, the dispersion relation is

$$
k_{0}^{2}=\vec{k}^{2}\left(1+2 c_{\gamma}+2\left(c_{\gamma}-c_{e}\right) \Pi_{\epsilon}\left(\vec{k}^{2}\right)\right),
$$

where

$$
\begin{aligned}
\Pi_{\epsilon}\left(\vec{k}^{2}\right)= & \frac{e^{2}}{2 \pi^{2}} \int_{0}^{1} d x x(1-x) \\
& \times \ln \left(1+2\left(c_{e}-c_{\gamma}\right) x(1-x) \frac{\vec{k}^{2}}{m^{2}}\right) .
\end{aligned}
$$

Let us introduce a notation $y \equiv\left(c_{e}-c_{\gamma}\right) \frac{\vec{k}^{2}}{m^{2}}$ and perform integration in (23) analytically. In the case $y>-2$, one obtains

$$
\Pi_{\epsilon}\left(\vec{k}^{2}\right)=\frac{e^{2}}{2 \pi^{2}}\left[\frac{y-1}{3 y} \sqrt{\frac{y+2}{y}} \operatorname{arcth} \sqrt{\frac{y}{y+2}}+\frac{1}{3 y}-\frac{5}{18}\right]
$$

otherwise $(y<-2)$, the polarization operator gains a nonzero imaginary part. According to the optical theorem, the process of photon decay to an electron-positron pair $\gamma \rightarrow e^{+} e^{-}$takes place in this case. ${ }^{3}$ This process is extremely fast [14], so any phenomenological consideration of the modified dispersion relation seems to be irrelevant.

Let us go back to the case $y>-2$, in which the photon decay is kinematically forbidden. The expression (24) can be simplified in two limiting cases. In the limit of large positive $y \gg 1$, one obtains $\Pi_{\epsilon}=\frac{q^{2}}{12 \pi^{2}}\left(\ln (2 y)-\frac{5}{3}\right)$ [cf. (23)]. Then, the photon dispersion relation obtains a logarithmic correction:

\footnotetext{
${ }^{3}$ The condition $y=-2$, which determines the position of the logarithmic cut at the momentum complex plane, coincides with an energy threshold condition for the photon decay process.
}

$$
\begin{aligned}
k_{0}^{2}= & \vec{k}^{2}\left[1+2 c_{\gamma}+\frac{e^{2}}{6 \pi^{2}}\left(c_{\gamma}-c_{e}\right) \cdot\left[\ln \left(2\left(c_{e}-c_{\gamma}\right) \frac{\vec{k}^{2}}{m^{2}}\right)-\frac{5}{3}\right]\right], \\
& \left(c_{e}-c_{\gamma}\right) \frac{\vec{k}^{2}}{m^{2}} \gg 1 .
\end{aligned}
$$

The radiative correction of the photon dispersion relation results in the dependence of the photon velocity on its energy. Hence, the physical velocity, defined as $c_{\gamma}^{p h} \equiv \frac{\partial k_{0}}{\partial|\vec{k}|}$ [see (22) and (23)], is no longer a constant. In the limit $y \gg 1$, the physical photon velocity obtains a negative radiative correction:

$c_{\gamma}^{p h}=1+c_{\gamma}-\frac{e^{2}}{6 \pi^{2}} \cdot\left(c_{e}-c_{\gamma}\right) \cdot \ln \left(2\left(c_{e}-c_{\gamma}\right) \frac{\vec{k}^{2}}{m^{2}}\right)$.

The expression (26) coincides with the result for renormalization group analysis for $c_{\gamma}$ obtained in Ref. [18] if we take the renormalization group scale $\mu$ in Ref. [18] as $\mu=\sqrt{c_{e}-c_{\gamma}} E_{\gamma}$. This can be explained in the following way. Let us set $c_{e}=0$ [this can be achieved via the field and coordinate redefinition; see the footnote after formula (6)]. The photon polarization operator (16) considered on shell may be interpreted as an off-shell polarization operator calculated in LI theory with the squared photon momentum

$$
q^{2} \equiv E_{\gamma}^{2}-\vec{k}^{2}=2\left(c_{\gamma}-c_{e}\right) E_{\gamma}^{2}
$$

The case of the logarithmic correction $y \gg 1$ corresponds to $q^{2} \gg m^{2}$.

In the opposite limit $|y| \ll 1$, the expression (22) can be expanded into series in $y$, and the leading term is $\Pi_{\epsilon}=\frac{e^{2}}{30 \pi^{2}} y+O\left(y^{2}\right)$. The effective photon dispersion relation (22) acquires an extra quartic term in the leading order:

$k_{0}^{2}=\vec{k}^{2}\left(1+2 c_{\gamma}\right)-\frac{\vec{k}^{4}}{M_{L V, e}^{2}}, \quad\left|c_{e}-c_{\gamma}\right| \frac{\vec{k}^{2}}{m^{2}} \ll 1$.

Here, the effective LV mass scale $M_{\mathrm{LV}, e}$ is defined as

$$
M_{\mathrm{LV}, e}=\frac{\sqrt{15} \pi}{e} \cdot \frac{m}{\left|c_{e}-c_{\gamma}\right|} .
$$

Let us note that the minus sign before the quartic term in (28) appears for both positive and negative $y$. The next-toleading term in (28) is expected to be of the order $O\left(\left(c_{\gamma}-c_{e}\right)^{-1} \frac{\vec{k}^{6}}{M_{\mathrm{LV}, e}^{4}}\right),{ }^{4}$ and it may take a plus or minus sign depending on the sign of $\left(c_{\gamma}-c_{e}\right)$. Similarly to the previous case, the physical photon velocity depends on its

\footnotetext{
${ }^{4}$ However, this term is of the order of $\alpha_{e m}^{2}$, as well as the contribution from two-loop correction to the polarization operator.
} 
momentum: $c_{\gamma}^{p h}=1+c_{\gamma}-\frac{3 \vec{k}^{2}}{2 M_{\mathrm{LV} . e}^{2}}$. This dependence may be tested experimentally, which we study in the next section.

\section{EXPERIMENTAL CONSTRAINTS ON LV IN FERMION SECTOR FROM PHOTON OBSERVATIONS}

In the previous section, we have calculated the radiative correction to the photon dispersion relation in QED, considering an electron running in the loop in the photon polarization operator. However, in the full Standard Model, the photon polarization operator in fact gets corrections not only from electrons but from all charged particles present in the theory. Assuming tree-level LV for a certain charged particle, one can perform machinery similar to the aforementioned one and obtain the radiative correction to the photon velocity caused by this particle. For two or more particles with a nonzero analog of $c_{e}$ (electric charges are assumed to be the same), in the first order on $\alpha_{e m}$, the full correction to the photon dispersion relation is the sum of corrections calculated for corresponding particles. For two or more quartic corrections, associated with charged fermions, the effective LV mass scale (29) is determined as

$$
M_{\mathrm{LV}}^{-2}=\sum_{f} M_{\mathrm{LV}, f}^{-2}
$$

Here, we summed over all charged fermions $f$, and $M_{\mathrm{LV}, f}$ is defined by the formula (29) for a concrete charged fermion with maximal tree-level velocity $1+c_{f}$, electric charge $e_{f}$, and mass $m_{f}$. If $M_{\mathrm{LV}, f}^{-2}$ for a certain fermion significantly exceeds the same parameter for other charged fermions, we can set $M_{\mathrm{LV}} \simeq M_{\mathrm{LV}, f}$ with a good accuracy. Thus, we can treat LV coefficients $c_{f}$ for different charged fermions separately.

Let us turn to the experimental constraints. The best direct constraints on the photon velocity are based on photon time-of-flight analysis for fast distant astrophysical sources. Thus, in the presence of LV, characterized by the dispersion relation (28), high-energy photons from a source would arrive later than low-energy ones. The best constraint of this type [25] is based on timing of the GRB 090510 event, observed by FERMI-LAT [26]. In the analysis [25], the quartic dispersion relation (28) has been tested for photon energies up to $150 \mathrm{MeV}$, and the lower bound on the $\mathrm{LV}$ mass scale $M_{\mathrm{LV}}=M_{\mathrm{LV}}^{\mathrm{GRB}} \equiv 1.3 \times 10^{11} \mathrm{GeV}$ was established at $95 \%$ C.L. In other words, $M_{\mathrm{LV}}$ calculated by formulas (29) and (30) should exceed $M_{\mathrm{LV}}^{\mathrm{GRB}}$. Taking into account the expression for $M_{\mathrm{LV}}$ for a certain fermion $f$, we obtain

$\left|c_{f}-c_{\gamma}\right|<\frac{\sqrt{15} \pi}{e_{f}} \cdot \frac{m_{f}}{M_{\mathrm{LV}}^{\mathrm{GRB}}} \simeq 3 \times 10^{-10} \cdot\left(\frac{e_{f}}{e}\right)^{-1} \cdot\left(\frac{m_{f}}{\mathrm{GeV}}\right)$.
TABLE I. Bounds on $\left|c_{f}-c_{\gamma}\right|$ for three generations of leptons.

\begin{tabular}{lcc}
\hline \hline & Our bound & Current bounds \\
\hline Electron & $1.5 \times 10^{-13}$ & $10^{-15}[27]$ \\
Muon & $3 \times 10^{-11}$ & $10^{-11}[28]$ \\
Tau lepton & $1.2 \times 10^{-9}$ & $10^{-8}[28]$ \\
\hline \hline
\end{tabular}

The formula (31) is valid under the condition $|y| \ll 1$, which leads to $\left|c_{f}-c_{\gamma}\right| \ll\left(m_{f} / 150 \mathrm{MeV}\right)^{2}$. This condition, combined with (31), is satisfied at least for leptons.

\section{A. Bounds in lepton sector}

In Table I, we present bounds on the value $\left|c_{f}-c_{\gamma}\right|$, where $c_{f}$ rely on three generations of leptons. Comparison with the current bounds [11] for each particle is also presented in Table I. Our bound for the electron is weaker than the current one [27], obtained from the absence of anomaly synchrotron losses at large electron-positron collider. However, for heavy leptons, the situation changes. The bound for the muon is of the same order as the current one; the bound on the tau lepton is 1 order of magnitude better. Since the bound on $\left|c_{f}-c_{\gamma}\right|$ for an electron is significantly better than for heavy leptons, the muon and tau bounds from Table I may be considered as bounds only on $\left|c_{\mu}\right|$ and $\left|c_{\tau}\right|$. We do not consider the case of fine-tuning $\left|c_{e}-c_{\gamma}\right| \ll c_{\gamma}$ here.

\section{B. QCD sector: The bound on $c_{f}$ for top quark}

The full photon polarization operator includes the contribution from the QCD sector as well. The following issue arises: should we work in the perturbative regime and consider quarks running in the loop or work in the nonperturbative regime and consider the effective theory?

Following the analogy with the off-shell polarization operator (see the end of the previous section), we compare the "transferred momentum" $q^{2} \equiv E_{\gamma}^{2}-\vec{k}^{2}$ with $\Lambda_{\mathrm{QCD}}^{2}$ : at large $q^{2} \gg \Lambda_{\mathrm{QCD}}^{2}$, the QCD corrections are perturbative and small; in the opposite case $q^{2} \ll \Lambda_{\mathrm{QCD}}^{2}$, the perturbative treatment is not applicable. Using (27), let us rewrite the condition for the perturbative regime as

$$
\left|c_{q}\right| \gg \frac{1}{2} \frac{\Lambda_{\mathrm{QCD}}^{2}}{E_{\gamma}^{2}} .
$$

Here, $c_{q}$ refers to the parameter $c_{f}$ for quarks. The energy scale of gamma-ray burst (GRB) bound $E_{\gamma}^{\mathrm{GRB}}=150 \mathrm{MeV}$ [25] is too small to make any bounds for quarks. Let us take another timing constraint from the flare of active galaxy PKS 2155-304 [29], which is a bit weaker than the GRB bound [25] but based on the observation of more energetic photons. The analysis of the flare performed by the H.E.S.S. Collaboration [29] set the bound $M_{\mathrm{LV}}>6.4 \times 10^{10} \mathrm{GeV}$; 
photons with energies $E_{\gamma} \sim 0.25-4 \mathrm{TeV}$ (mean energy $1 \mathrm{TeV}$ ) were considered. For these energies, the condition (32) takes the numerical value

$$
\left|c_{q}\right| \gg 2.4 \times 10^{-8} \text {. }
$$

Here, the value $\Lambda_{\mathrm{QCD}} \approx 217 \mathrm{MeV}$ has been used.

For these values of $c_{q}$ and $E_{\gamma}$, the condition $|y| \ll 1$ may be valid only for the top quark. Performing an analysis similar to (31) with $M_{\mathrm{LV}}^{\mathrm{AGN}} \equiv 6.4 \times 10^{10} \mathrm{GeV}$ instead of $M_{\mathrm{LV}}^{\mathrm{GRB}}$ and the top-quark electric charge $e_{\text {top }}=2 / 3 e$, one arrives at the following bound for the parameter $c_{q}$ for the top quark:

$$
\left|c_{\text {top }}\right|<1.6 \times 10^{-7}
$$

The conditions (33), and $|y| \ll 1$, are valid for this bound. The bound (34) is 5 orders of magnitude better than the direct collider bound from the Tevatron [30] (see also the prospect for a collider bound for the LHC [31]).

For light quarks, this analysis fails; one should consider the case $y \gg 1$. The bounds on $c_{e}$ for light quarks and/or mesons may be a scope of a separate work and should be compared with collider bounds $[32,33]$.

\section{DISCUSSION}

We have calculated the finite momentum-dependent part of the photon polarization operator in a simple model of LV QED in the one-loop approximation, considering LV coefficients nonperturbatively. The components of the one-loop polarization operator are rescaled to the components of the LI one due to the presence of a single particle inside the loop. The modified photon propagator, obtained by the summation of one-particle reducible contributions to the photon polarization operator, has nontrivial poles which determine radiatively corrected dispersion relations. In different regimes, the correction is either quartic on momentum or logarithmic; the physical velocity for a free photon acquires radiative corrections in the corresponding way. The logarithmic correction to the photon velocity coincides with the result of renormalization group analysis for the corresponding coefficient, obtained in Ref. [18] using infinite parts of one-loop diagrams. The reason of it is that the on-shell squared momentum for the LV photon may be considered as off-shell squared momentum for the LI polarization operator, which is the standard interpretation of the renormalization group scale.

The position of the logarithmic cut in the momentum plane is shifted compared to the standard case, in accordance with the optical theorem. This effect seems to be taken into account only for nonperturbative treatment of LV parameters and is usually missed in perturbative calculations (see Ref. [19]).
Radiative corrections to the photon velocity, induced by a loop of a charged particle with tree-level LV, can be tested experimentally. The corresponding observations constrain LV for all charged fermions, and the bounds for the $\tau$ lepton and top quark are the best in the literature. In any case, no charged fermion can have large values of $c_{e}$; otherwise, the photon velocity would strongly depend on its energy (or the photon would decay).

It turns out that only the region $y \ll 1$ is relevant for phenomenology. However, this fact was not obvious before the calculation. One can imagine some physical situations in which $y$ may be larger than unity. First, consider more energetic photons. Let us note that $y$ grows with the photon energy. At photon energies $\sim 50 \mathrm{TeV}$, the contribution to $y$ from muons may be of the order of unity (the value $c_{\mu} \sim 10^{-11}$ is not restricted). At these energies, there are no photon timing measurements, so full analysis including arbitrary $y$ seems to be necessary. A similar situation would arise if more energetic photons $\left(10^{14}-10^{19} \mathrm{eV}\right)$ were to be detected. A more exotic example is a hypothetical millicharged fermion [34] inside the loop. For this type of particle, the regime $y \gg 1$ would be relevant as well.

The modified dispersion relation for a free photon acquires novel momentum-dependent terms, which are absent in tree-level dispersion relation (5). This fact may be also shown in terms of the effective Lagrangian for a photon. Integrating out charged fermions with tree-level LV, one should obtain an addition to the effective Lagrangian, which in the lowest order is expected to be equal to

$$
\delta \mathcal{L}_{\text {eff }} \sim-\frac{1}{4 M_{\mathrm{LV}}^{2}} F_{i j} \Delta F^{i j}
$$

Here, $M_{\mathrm{LV}}$ is determined according formula (30) for LV charged fermions. We can integrate out all charged Standard Model particles except the electrons and obtain the QED effective Lagrangian which includes the dimension-6 kinetic term (35). Such high-dimensional terms in the LV QED Lagrangian have been considered in the literature (see Refs. [14,24,35] for example). In these models, QED Feynman rules are modified, so there are some changes in thresholds and cross sections for several tree-level processes in QED. Astrophysically relevant examples of such processes are pair production by a high-energy photon on photon background or in the Coulomb field [14]. These reactions influence the processes of photon propagation in the extragalactic medium and shower formation in the atmosphere. Experimental constraints on $M_{\mathrm{LV}}$, based on the detection of TeV photons from astrophysical sources, are of the same order but a bit better than $M_{\mathrm{LV}}^{\mathrm{GRB}}$ [36]. Considerations of these processes may set a bit stronger bounds on the parameter $c_{f}$ for 
charged fermions than presented in Table I. Hypothetical experimental observation of ultrahigh-energy $\left(\sim 10^{19} \mathrm{eV}\right)$ photons would establish significantly better constraints on $M_{\mathrm{LV}}$ [37] and subsequently better constraints on $c_{f}$.

Radiative corrections to the physical velocity can be considered nonperturbatively on $c_{e}$ and $c_{\gamma}$ for electrons as well. Such calculation, perturbative on SME parameters, has been performed in Ref. [19]. Nonperturbative calculation in our simplified model can be a good test of it. The radiative corrections to the electron velocity can be tested experimentally as well. However, the corresponding constraints are expected to be worse than the constraints from photon velocity measurements.

\section{ACKNOWLEDGMENTS}

The author is thankful to Sergey Sibiryakov, Dmitry Kirpichnikov, Dmitry Gorbunov, Emin Nugaev, Ivan Kharuk, and Grigory Rubtsov for helpful discussions and comments on the draft of the paper. This work was supported by RSF Grant No. 14-22-00161.

\section{APPENDIX: RADIATIVELY CORRECTED PHOTON PROPAGATOR IN PSEUDO-LORENTZ GAUGE}

Let us summarize one-particle reducible contributions to the photon propagator in pseudo-Lorentz gauge (9). It is simpler to perform field and coordinate rescaling (see footnote 2) in order to set $c_{\gamma}=0$ (otherwise, a complicated resummation should be needed). The summation yields

$$
\begin{aligned}
\Delta_{\mu \nu}(k)= & \frac{1}{1-\Pi\left(\hat{k}^{2}\right)\left(1-c_{e}\right)} \\
& \times\left[\frac{\left(P_{1}-P_{2}\right)_{\mu \nu}}{k_{0}^{2}-\vec{k}^{2}}+\frac{\left(P_{2}\right)_{\mu \nu}}{k_{0}^{2}-\vec{k}^{2}\left(1-2 c_{e} \Pi\left(\hat{k}^{2}\right)\right)}\right],
\end{aligned}
$$

where projectors $P_{1}^{\mu \nu}$ and $P_{2}^{\mu \nu}$ were defined in (17). The overall coefficient $1 /\left[1-\Pi\left(\hat{k}^{2}\right)\left(1-c_{e}\right)\right]$ determines the Landau pole, as previously. The position of the pole of the second term gives the photon dispersion relation. At first sight, it seems that the propagator (A1) describes more degrees of freedom because of another pole in the first term corresponding to a relativistic dispersion relation. This would contradict the fact that photons have two polarizations that, according to $C P T$, must propagate with the same velocity. In fact, the relativistic pole is a pure gauge artifact and disappears from gauge invariants. Indeed, consider the photon exchange amplitude between two conserved currents:

$$
\mathcal{A}=J_{1}^{\mu} \Delta_{\mu \nu}(k) J_{2}^{\nu}, \quad k_{\mu} J_{1}^{\mu}=k_{\mu} J_{2}^{\mu}=0 .
$$

A straightforward calculation yields

$\mathcal{A}=\frac{1}{1-\Pi\left(\hat{k}^{2}\right)\left(1-c_{e}\right)} \cdot \frac{\left(1-2 c_{e} \Pi\left(\hat{k}^{2}\right)\right) J_{1}^{0} J_{2}^{0}-J_{1}^{i} J_{2}^{i}}{k_{0}^{2}-\vec{k}^{2}\left(1-2 c_{e} \Pi\left(\hat{k}^{2}\right)\right)}$.

We see that the spurious pole has completely disappeared, and (A3) gives the modified dispersion relation (22).
[1] F. Girelli, F. Hinterleitner, and S. Major, SIGMA 8, 098 (2012).

[2] I. Mocioiu, M. Pospelov, and R. Roiban, Phys. Lett. B 489, 390 (2000).

[3] J. R. Ellis, N. E. Mavromatos, D. V. Nanopoulos, and A. S. Sakharov, Int. J. Mod. Phys. A 19, 4413 (2004); N.E. Mavromatos, Int. J. Mod. Phys. A 25, 5409 (2010).

[4] V. A. Kostelecky and S. Samuel, Phys. Rev. D 39, 683 (1989).

[5] P. Horava, Phys. Rev. D 79, 084008 (2009).

[6] D. Blas, O. Pujolas, and S. Sibiryakov, Phys. Rev. Lett. 104, 181302 (2010); J. High Energy Phys. 04 (2011) 018.

[7] D. Mattingly, Living Rev. Relativity 8, 5 (2005).

[8] S. Liberati, Classical Quantum Gravity 30, 133001 (2013).

[9] G. Amelino-Camelia, Living Rev. Relativity 16, 5 (2013).

[10] D. Colladay and V. A. Kostelecky, Phys. Rev. D 58, 116002 (1998).

[11] V. A. Kostelecky and N. Russell, Rev. Mod. Phys. 83, 11 (2011).
[12] V. A. Kostelecky and R. Lehnert, Phys. Rev. D 63, 065008 (2001).

[13] D. Colladay and V. A. Kostelecky, Phys. Lett. B 511, 209 (2001).

[14] G. Rubtsov, P. Satunin, and S. Sibiryakov, Phys. Rev. D 86, 085012 (2012).

[15] S. R. Coleman and S. L. Glashow, Phys. Lett. B 405, 249 (1997); Phys. Rev. D 59, 116008 (1999).

[16] T. Jacobson, S. Liberati, and D. Mattingly, Phys. Rev. D 67, 124011 (2003); Ann. Phys. (Amsterdam) 321, 150 (2006).

[17] S. Chadha and H. B. Nielsen, Nucl. Phys. B217, 125 (1983).

[18] V. A. Kostelecky, C. D. Lane, and A. G. M. Pickering, Phys. Rev. D 65, 056006 (2002).

[19] M. Cambiaso, R. Lehnert, and R. Potting, Phys. Rev. D 90, 065003 (2014).

[20] R. Potting, Phys. Rev. D 85, 045033 (2012).

[21] A. Kuznetsov and N. Mikheev, Springer Tracts Mod. Phys. 252, 1 (2013).

[22] T. J. Hollowood, G. M. Shore, and R. J. Stanley, J. High Energy Phys. 08 (2009) 089. 
[23] G. de Berredo-Peixoto and I. L. Shapiro, Phys. Lett. B 642, 153 (2006).

[24] D. Anselmi and M. Taiuti, Phys. Rev. D 83, 056010 (2011).

[25] V. Vasileiou, A. Jacholkowska, F. Piron, J. Bolmont, C. Couturier, J. Granot, F. W. Stecker, J. Cohen-Tanugi, and F. Longo, Phys. Rev. D 87, 122001 (2013).

[26] M. Ackermann et al., Astrophys. J. 716, 1178 (2010).

[27] B. Altschul, Phys. Rev. D 82, 016002 (2010).

[28] B. Altschul, Astropart. Phys. 28, 380 (2007).

[29] A. Abramowski et al. (HESS Collaboration), Astropart. Phys. 34, 738 (2011).

[30] V. M. Abazov et al. (D0 Collaboration), Phys. Rev. Lett. 108, 261603 (2012).
[31] M. S. Berger, V. A. Kostelecký, and Z. Liu, Phys. Rev. D 93, 036005 (2016).

[32] V. A. Kostelecký, E. Lunghi, and A. R. Vieira, Phys. Lett. B 769, 272 (2017).

[33] I. Karpikov, D. Kirpichnikov, and D. Tlisov, arXiv: 1612.02217.

[34] A. Y. Ignatiev, V. A. Kuzmin, and M. E. Shaposhnikov, Phys. Lett. 84B, 315 (1979); L. B. Okun, M. B. Voloshin, and V. I. ZakharovPhys. Lett. 138B, 115 (1984).

[35] D. Mattingly, arXiv:0802.1561.

[36] G. Rubtsov, P. Satunin, and S. Sibiryakov, J. Cosmol. Astropart. Phys. 05 (2017) 049.

[37] G. Rubtsov, P. Satunin, and S. Sibiryakov, Phys. Rev. D 89, 123011 (2014). 